\title{
Publisher Correction: Exploring wet markets
}

Correction to: Nature Food https://doi.org/10.1038/s43016-020-0090-1, published online 19 May 2020.

In the version of this Editorial originally published, the word 'cull' and its derivatives was used incorrectly instead of 'slaughter' and its derivatives. Three sentences beginning "Most sell vegetables..., "However, the calls to ban wet markets..." and "Recent portrayals of wet markets..." have now been amended in all versions of the Editorial.

Published online: 22 May 2020

https://doi.org/10.1038/s43016-020-0099-5

๑) Springer Nature Limited 2020 